Proceedings of the 2011 Winter Simulation Conference

S. Jain, R. R. Creasey, J. Himmelspach, K. P. White, and M. Fu, eds.

\title{
A TWO-STAGE NON-LINEAR PROGRAM FOR OPTIMAL ELECTRICAL GRID POWER BALANCE UNDER UNCERTAINTY
}

\author{
Dzung Phan \\ Soumyadip Ghosh
}

IBM T.J. Watson Research Center

P.O. Box 218, 1101 Kitchawan Rd.

Yorktown Heights NY 10598, USA

\begin{abstract}
We propose a two-stage non-linear stochastic formulation for the economic dispatch problem under renewable-generation uncertainty. Each stage models dispatching and transmission decisions that are made on subsequent time periods. Certain generation decisions are made only in the first stage and the second stage realizes the actual renewable generation, where the uncertainty in renewable output is captured by a finite number of scenarios. Any resulting supply-demand mis-match must then be alleviated using extra, high marginal-cost power sources that can be tapped in short order. We propose two outer approximation algorithms to solve this nonconvex optimization problem to optimality. We show that under certain conditions the sequence of optimal solutions obtained under both alternatives has a limit point that is a globally-optimal solution to the original two-stage nonconvex program. Numerical experiments for a variety of parameter settings were carried out to indicate the efficiency and usability of this method of large practical instances.
\end{abstract}

\section{INTRODUCTION}

With the advent of the Smart Grid, the infrastructure for energy supply generation and transmission is experiencing a transition from the current centralized system to a decentralized one. The responsiveness and flexibility envisioned for the Smart Grid provides additional advantages in facing the significant new challenges (Cheung et al. 2010, Li et al. 2007) of integrating distributed and intermittent generation capability, such as small generators and renewable energy sources (wind, solar, etc.), at a scale that current grid technology is finding hard to achieve. This is becoming more critical as renewable energy technologies are playing an increasingly visible role in the portfolio mix of electricity generation.

Current practice does planning for energy supply generation and supply-demand mediation in a power grid in two steps. The first step takes place in a day-ahead market and decides which bulk generation sources (typically thermal, nuclear and hydro sources) are awarded contracts to supply energy in the next day. This base generation capability is augmented by additional smaller capacity "peaker" thermal generators and external sources of energy (spot markets) connected to a subset of the grid's nodes, to hedge against un-planned excess demand. The second planning stage, which is the problem of this paper's interest, is at a smaller time-scale, typically five to fifteen minutes. It decides how the active generators are dispatched (e.g., set the level at which bulk generators produce energy) and how the produced energy is routed through the grid to consumption (or load) nodes. Transmission occurs across multiple buses (network nodes) interconnected via electrical transmission lines, and the flow obeys certain non-linear equations that arise out of Kirchoff's laws (see e.g. I.P.E. Society (1996) for a review). An economic dispatch (ED) problem or optimal power flow (OPF) problem is said to have been solved when the dispatch and transmission decisions are taken to minimize the total cost of generation (Carpentier 1962). 


\section{Phan and Ghosh}

We consider a stochastic economic dispatch problem where we introduce a renewable resource of energy to the grid. To keep the discussion tractable, we shall consider only wind generation as the main renewable resource in this paper, though the methods developed here apply transparently to all renewables. Wind generation has negligible operational costs (in the hourly time-scale) and thus should be the first generator to be dispatched. Indeed, regulations in multiple US states require the use of wind power if it is being generated. However, the intermittent nature of output from wind turbines due to weather conditions is often seen as a potential obstacle to dispatching wind power in the classical sense. Hence, we model the wind power as a non-dispatchable, variable generation source that is connected in an always-on state to the grid. Current levels of penetration of renewable generation makes the scenario of over-supply because of renewables a very remote possibility.

Forecasting near-term wind availability and velocity is an imperfect science with significant variability between the forecast and the realized generation. In Dragoon and Milligan (2003), the authors consider integrating wind power production into existing dispatch models, and analyze the uncertainty of forecast errors for wind power production and its impact on incremental reserve requirements and imbalance costs. The agency charged with controlling the smooth operation of the grid will require that this uncertainty associated with utilizing non-renewable sources be hedged against. This problem is often addressed by balancing energy provided by non-dispatchable sources (such as wind and photovoltaic units) with quickly dispatchable, albeit costly, sources (such as small hydro and micro turbine units). This problem has been studied in various levels of sophistication starting from individual end-users up to local utilities. In particular, a balancing approach to achieve overall dispatchability in a distributed generation network is presented in Xue et al. (2007), which consequently converts a group of small distributed generations into a large logical generation station.

Another stream of research incorporates the uncertainty in setting or adjusting the dispatch and transmission decisions, which has the effect of dispatching additional capacity to hedge against the risk of a large unforeseen shortfall in total supply. The study in Brini et al. (2009) considers an economic environmental dispatching model where wind and solar energy are both included but constrained to be no more than $30 \%$ of the total dispatched capacity. Hatami et al. (2009) propose a stochastic programming framework to determine the optimal procurement of interruptible load in order to minimize the risk of a shortfall over multiple periods. The stochastic OPF can be solved by imposing a set of risk constraints, in the form of chance constraints in Fu and McCalley (2001) or mean-excess constraints in Ghosh et al. (2011), to balance risk of shortfalls due to uncertain generation against cost of provisioning corrective generation sources such a peakers.

In this paper, we propose a two-stage stochastic formulation to address the wind-generation uncertainty. The two stages model dispatching and transmission decisions that are made on subsequent time periods separated by say fifteen minutes or an hour. Our main point of departure from current literature is in our consideration of the full non-linear power balance equations in each stage of this program. Certain dispatching decisions cannot be changed in the subsequent step; for example, mechanical stability considerations require that large diesel/coal generators change their generation levels more gradually. Hence, these decisions are made in the first stage and remain fixed at the second stage. (More generally, one may allow these to be varied in a small interval around their first stage values, and we anticipate our findings will hold in this case.) The second stage realizes the actual wind generation (or is only minutes away from realizing it and thus has a much more accurate forecast), where the uncertainty in wind power output is captured by a finite number of scenarios. Any resulting supply-demand mis-match must then be alleviated using extra, high marginal-cost power sources that can be tapped in short order. We call this second class of generators collectively as the spot-market, which can include a) thermal "peakers", which are active, quick-response fossil-fuel sources, b) external power sources such as neighboring grids or power aggregators that are willing to supply extra power at "spot market" prices, and c) sources of virtual generation, such as interruptible loads of large commercial users, retail operations or consumer homes that can be influenced to shift or reduce their demand in response to incentives (refer Ghosh et al. (2010) for further details). 


\section{Phan and Ghosh}

This two-stage stochastic program is non-linear due to the Kirchoff's law based power transmission equations, and are generally non-convex. Non-convex two-stage programs are hard to solve using standard techniques, and the literature has little to offer as a general prescription. The key to solving such problems seems to be the ability to exploit structural properties of a specific formulation. Lavaei and Low (2010) give such a strong structural property for the standard economic dispatch problem (i.e., the single stage deterministic ED problem), where they show that if the problem instance satisfies a certain reasonable condition then one can obtain provably-global optimal solutions to the problem. We make use of this property to provide an approach to solving this two-stage problem.

Specifically, our contributions are:

- We show that for problem instances that satisfy the condition given by Lavaei and Low (2010), an effective, consistent decomposition scheme can be setup for the two-stage problem, much like the standard decomposition for two-stage linear programs. In particular, this facilitates a parallel implementation of the solution algorithm.

- The decomposition scheme solves a sequence of lower-approximation problems for the first-stage minimization problem. Each iteration improves a piecewise-linear outer-approximation for the recourse function that represents the second stage costs of the first stage decisions.

- We propose two alternatives for the form of the outer-approximation. We show that the sequence of optimal solutions obtained under both alternatives have a limit point that is a globally-optimal solution to the original two-stage non-convex program.

- We performed numerical experiments for a variety of parameter settings that indicate the efficiency and usability of this method of large practical instances.

The remainder of the paper is organized as follows. Section 2 introduces both our model notation and formulation. Section 3 presents the decomposition and outer-approximation schemes and the proofs of global-convergence. Section 4 presents the numerical experiments and analysis, followed by some concluding remarks.

\section{MODEL DESCRIPTION}

An electric grid management entity controls the dispatching of active generation units and the spot market over a network of multiple local buses interconnected via transmission lines. Define as $\mathcal{B}$ the set of all buses, or nodes, in the grid network, $\mathcal{G}$ as the set of buses that conventional generators connect to, $\mathcal{W}$ as the set of wind-generator buses, $\mathcal{D}$ the set of energy demand or load buses, and $\mathcal{S}$ as the set of buses with access to the spot market. To keep the exposition readable, we will assume that the sets $\mathcal{G}, \mathcal{W}, \mathcal{D}$ and $\mathcal{S}$ are pairwise disjoint, and $\mathcal{B}=\mathcal{G} \cup \mathcal{W} \cup \mathcal{D} \cup \mathcal{S}$.

Power at any bus $i$ is represented by a complex number $P_{i}+\mathfrak{i} Q_{i}$, where $P_{i}$ and $Q_{i}$ are the active and reactive parts, and $\mathfrak{i}$ is the imaginary unit. Power demand is represented by $P_{i}^{d}$ and $Q_{i}^{d}$, while $P_{i}^{g}$ and $Q_{i}^{g}$ are the power extracted from conventional generators. In our formulation, the first stage generation decisions are represented by $P_{i}^{q}+\mathfrak{i} Q_{i}^{g}$, and the cost of generation is given by the convex quadratic function:

$$
f\left(P^{g}\right) \triangleq \sum_{i \in \mathcal{G}}\left(c_{0 i}\left(P_{i}^{g}\right)^{2}+c_{1 i} P_{i}^{g}+c_{2 i}\right),
$$

where $c_{0 i}, c_{1 i}$ and $c_{2 i}$ are nonnegative parameters. We define by $P^{g}$ the vector whose components are $P_{i}^{g}$ 's, $i \in \mathcal{G}$; similar vectors for related quantities will be considered in the same manner. The generators are limited to producing within $\left[P^{\min }, P^{\max }\right]$ and $\left[Q^{\min }, Q^{\max }\right]$, the active and reactive limits respectively. Scenario $s \in \mathcal{P}$ of the second stage realizes $P_{i}^{w, s}+\mathfrak{i} Q_{i}^{w, s}$ of power extraction from wind generator at bus $i$. Each scenario $s$ has a probability of occurrence $p^{s}$. The second stage recourse variables are the extra power $\left(P_{i}^{i n, s}, Q_{i}^{i n, s}\right)$ that may be purchased from the spot market at bus $i$ under scenario $s$, allowing for the possibility that surplus power $\left(P_{i}^{\text {out }, s}, Q_{i}^{\text {out }, s}\right)$ may also be sold to the spot market. Cost curve $g^{s}\left(P^{i n, s}\right)$ 


\section{Phan and Ghosh}

gives the total cost of purchasing from the spot market under scenario $s$, while $h^{s}\left(P^{\text {out,s}}\right)$ represents the total revenue generated by selling to the spot market. In our numerical experiments, we assume $g^{s}$ is convex and quadratic and $h^{s}$ is linear. We allow these options to be exercised in the first-stage too; typical costs $f$, $g^{s}, h^{s}$, and available generation capacity are such that the first-stage should never result in a spot-market purchase.

Power transmission over a network of transmission lines that connect all the buses is determined by the voltages set at each bus. Let $\boldsymbol{V}$ be the vector of voltages $V_{i}$ at all buses. Then, various physical and safety considerations require that $|V| \in\left[V^{\min }, V^{\max }\right]$. Current flow between any two connected nodes $i$ and $j$ is determined by the voltage difference between them as $I_{i j}=Y_{i j}\left(V_{j}-V_{i}\right)$, where the admittance $Y_{i j}$ between nodes $i$ and $j$ is a physical characteristic of the network. In short, we have $\boldsymbol{I}=Y \cdot \boldsymbol{V}$, where $Y$ is the admittance matrix. Net power flow between nodes $i$ and $j$ is then determined as $\boldsymbol{V} \cdot \boldsymbol{I}^{*}$, where $\boldsymbol{I}^{*}$ is the complex conjugate of $\boldsymbol{I}$. Collectively represent all the net active and reactive power flows into node $i$ by $P F_{i}(V)$ and $Q F_{i}(V)$ respectively. Each function is quadratic in the real and imaginary parts of the base transmission variables $V$, and in general is non-convex. With a small abuse of notation, let $s=0$ represent the first stage. Then the following balance equations are implied for active and reactive power (for all $s \in 0 \cup \mathcal{P}$ ):

$$
P F_{i}^{s}\left(V^{s}\right)=\left\{\begin{array}{ll}
P_{i}^{i n, s}-P_{i}^{\text {out }, s} & \forall i \in \mathcal{S} \\
P_{i}^{g} & \forall i \in \mathcal{G} \\
P_{i}^{w, s} & \forall i \in \mathcal{W} \\
-P_{i}^{d} & \forall i \in \mathcal{D}
\end{array} \quad Q F_{i}^{s}\left(V^{s}\right)= \begin{cases}Q_{i}^{i n, s}-Q_{i}^{\text {out }, s} & \forall i \in \mathcal{S} \\
Q_{i}^{g} & \forall i \in \mathcal{G} \\
Q_{i}^{w, s} & \forall i \in \mathcal{W} \\
-Q_{i}^{d} & \forall i \in \mathcal{D}\end{cases}\right.
$$

The objective of the two-stage optimal power flow problem is to minimize, over the variables $P_{i}^{g}, Q_{i}^{g}, V_{i}^{s}$, $P_{i}^{i n, s}, P_{i}^{\text {out }, s}, Q_{i}^{i n, s}$ and $Q_{i}^{\text {out }, s}$, the total aggregated expected costs:

$$
\begin{aligned}
& \min f\left(P^{g}\right)+\sum_{s \in \mathcal{P}} p^{s}\left(g^{s}\left(P^{i n, s}\right)-h^{s}\left(P^{\text {out }, s}\right)\right) \\
& \text { s.t. } \quad \text { power flow constraints (1) } \\
& P_{i}^{\text {min }} \leq P_{i}^{g} \leq P_{i}^{\text {max }} \forall \quad i \in \mathcal{G} \\
& Q_{i}^{\text {min }} \leq Q_{i}^{g} \leq Q_{i}^{\text {max }} \forall i \in \mathcal{G} \\
& V_{i}^{\text {min }} \leq\left|V_{i}^{s}\right| \leq V_{i}^{\text {max }} \forall i \in \mathcal{B}
\end{aligned}
$$

\section{ALGORITHMS}

For notational simplicity, all real-imaginary pairs $(P, Q) \in \mathbb{R}^{2 n}$ of the complex value $P+\mathfrak{i} Q$ will be denoted by the shorthand $P Q \in \mathbb{R}^{2 n}$ for the rest of the paper. For example, $P Q^{g}$ should be read as $P^{g}+\mathfrak{i} Q^{g}$. The nonconvex optimization (2-3) can be compactly cast in the classical two-stage form:

$$
\begin{array}{ll}
\min & \Psi\left(P Q^{g}\right)=f\left(P^{g}\right)+\sum_{s \in \mathcal{P}} p^{s} \omega^{s}\left(P Q^{g}\right) \\
\text { subject to } & \left(P Q^{g}, V^{0}, P Q^{\text {in, }, 0}, P Q^{\text {out }, 0}, P Q^{w, 0}\right) \in X,
\end{array}
$$

where the feasible region $X$ of the first-stage decision variables is defined by the constraints (1) and (3) associated with the first-stage $(s=0)$. Note that we assumed $P Q^{i n, 0}=P Q^{\text {out }, 0}=0$. The second stage $\operatorname{cost} \omega^{s}$ for all $s \in \mathcal{P}$ :

$$
\begin{aligned}
\omega^{s}\left(P Q^{g}\right)= & \min \quad g^{s}\left(P^{\text {in,s }}\right)-h^{s}\left(P^{\text {out }, s}\right) \\
\text { subject to } \quad & E^{s}\left(V^{s}\right)=\left(P Q^{g}\right) \\
& R^{s}\left(V^{s}, P Q^{\text {in,s}}, P Q^{\text {out }, s}, P Q^{w, s}\right) \leq 0 .
\end{aligned}
$$




\section{Phan and Ghosh}

The equalities $E^{s}(\cdot)$ arise from power balance equations (1), while the constraints $R^{s}(\cdot)$ include all other second-stage constraints from the original problem (2-3) that do not include $P Q^{g}$. In particular, we define

$$
E^{s}\left(V^{s}\right)=\left(P F_{i}^{s}\left(V^{s}\right)+\mathfrak{i} Q F_{i}^{s}\left(V^{s}\right): i \in \mathcal{G}\right) .
$$

We will present two outer approximation algorithms to solve the master problem (M) to optimality. The key idea is that, under certain reasonable assumptions, the recourse function $\omega^{s}\left(P Q^{g}\right)$ is convex in $P Q^{g}$ even though the subproblems $\left(\boldsymbol{S}^{s}\right)$ are non-convex. The epigraph of $\omega^{s}\left(P Q^{g}\right)$ will be iteratively approximated by a number of affine inequalities that are sub-gradients of the function generated from the second stage problems $\left(\boldsymbol{S}^{s}\right)$. The spirit of our algorithms is closely related to the methods that solve convex non-linear programming problems (Geoffrion 1972, Grothey et al. 1999, Ruszczýnski 2003) or mixed-integer non-linear programs (Duran and Grossmann 1986, Fletcher and Leyffer 1994, Bonami et al. 2008). The outer approximation algorithms we present is in line with the generalized Benders decomposition approach of Geoffrion (1972), and differs in the method that is utilized to construct the outer linearizations of $\omega^{s}\left(P Q^{g}\right)$. We use the optimal dual solution of the $\left(\boldsymbol{S}^{s}\right)$ to obtain a sub-gradient to $\omega^{s}\left(P Q^{g}\right)$, while the earlier approach merely obtains an outer cover from the optimal primal values $\left(P Q^{g}\right)$. The epigraph of the nonlinear function is approximated by the intersection of a collection of sets with a simpler structure, e.g, polyhedra in our case.

Our proposed algorithms rely on the following two properties that have been shown to be widely shared in practical power grid balance problems (Lavaei and Low 2010, Phan 2010).

Property 1 For every $P Q^{g} \in\left[P^{\min }, P^{\max }\right] \times\left[Q^{\min }, Q^{\max }\right]$, the subproblem $\left(\boldsymbol{S}^{\boldsymbol{s}}\right)$ is feasible.

Property 1 says that any value of the generation power output $\left(P Q^{g}\right)$ chosen in the first-stage admits a feasible solution for each $\left(\boldsymbol{S}^{s}\right)$ by buying (or selling) the deficiency (or surplus) of energy from (to) the spot market. In effect, the spot market has limitless capacity, either to import or export energy. This property is a reasonable assumption, for instance the "spot market" typically consists of more than one source. Our method uses this assumption to side-step the usual second-stage feasibility checking and cut-generation part of decomposition-based algorithms. We will thus not cut off any feasible sub-regions in the algorithm's iterations. For cutting-plane based approaches to solving non-linear problems, feasibility cuts (especially linear cuts) could prune sub-regions of the (non-convex) feasible domain containing the global optimum, yielding only a locally optimal solution (see, for example, Sahinidis and Grossmann 1991), and extra machinery is required to prevent this eventuality.

Define the function

$$
l(\lambda, \gamma)=\inf _{V^{s}, P Q^{i n, s}, P Q^{\text {out }, s}} g^{s}\left(P^{\text {in }, s}\right)-h^{s}\left(P^{\text {out }, s}\right)+\lambda^{\top} R^{s}\left(V^{s}, \ldots\right)+\gamma^{\top} E^{s}\left(V^{s}\right) .
$$

The parameters $\gamma$ and $\lambda$ are the Lagrangian coefficients of the constraint-sets $E^{s}$ and $R^{s}$ respectively. Then, the standard Lagrangian dual formulation associated with the subproblem $\left(\boldsymbol{S}^{\boldsymbol{s}}\right)$ is:

$$
\max _{\lambda \geq 0, \gamma} l(\lambda, \gamma)-\gamma^{\top} P Q^{g}
$$

Property 2 For every $P Q^{g} \in\left[P^{\min }, P^{\max }\right] \times\left[Q^{\min }, Q^{\max }\right]$, the optimal solution of the Lagrangian dual (4) of subproblem $\left(\boldsymbol{S}^{\boldsymbol{s}}\right)$ has a zero duality gap with the associated primal solution.

By exploiting the physical properties of transmission lines, Lavaei and Low (2010) showed that the classical OPF problem corresponding to a practical power system has an equivalent form that easily satisfies the zero-duality-gap condition, and a globally optimal solution for the classical OPF problem can be recovered from this equivalent form. This condition is satisfied for a range of power grid test instances including all IEEE benchmark systems (see Lavaei and Low 2010, Phan 2010), and is expected to hold for every practical power system. For more details, please refer to Theorem 1 and the algebraic and geometric studies provided in Lavaei and Low (2010). Property 2 says that the subproblem $\left(\boldsymbol{S}^{\boldsymbol{s}}\right)$ in the standard OPF form thus inherits this property. We now characterize the recourse function $\omega^{s}\left(P Q^{g}\right)$ associated with second-stage scenario $s \in \mathcal{P}$. 


\section{Phan and Ghosh}

Theorem 1 If Property 2 holds, then $\omega^{s}\left(P Q^{g}\right)$ is convex. Furthermore, if $\hat{\gamma}$ is the Lagrangian multiplier corresponding to the constraint $E^{s}(\cdot)$ from the subproblem $\left(\boldsymbol{S}^{s}\right)$, then $-\hat{\gamma}$ is a subgradient of $\omega^{s}$ at $\left(P Q^{g}\right)$.

Proof. The subproblem $\left(\boldsymbol{S}^{\boldsymbol{s}}\right)$ is a standard optimal power flow problem with the generation decisions limited to $P Q^{i n, s}$ and $P Q^{\text {out,s }}$. As shown in (Lavaei and Low 2010), the standard OPF has an equivalent form that has its dual as a semidefinite program:

$$
\begin{aligned}
\omega^{s}\left(P Q^{g}\right)=\max _{u, v} & u^{\top} P Q^{g}+v^{\top} c+r \\
\text { s.t. } & \sum u_{i} A_{i}+\sum v_{i} B_{i} \preceq C,
\end{aligned}
$$

where problem data (vectors $c, r$ and matrices $A_{i}, B_{i}, C$ ) are defined by the system parameters $P^{\min }, P^{\max }$, $Q^{\min }, Q^{\max }, V^{\min }, V^{\max }, Y, P Q^{w, s}$ and the coefficients of (convex quadratic) cost functions $g^{s}$ and $h^{s}$. The equality of $\omega^{s}\left(P Q^{s}\right)$ to the problem (SDP) utilizes the Property 2. From Boyd and Vandenberghe (2004), p.67, it suffices for the first part to show that the function $\alpha(t) \triangleq \omega^{s}\left(P Q^{g}+t \bar{d}\right)$, is convex with respect to $t$ for all $P Q^{g}$ and $\bar{d}$. Indeed, let $t_{1}, t_{2} \in \operatorname{dom}(\alpha), \beta \in[0,1]$ and $\Omega=\left\{(u, v): \sum u_{i} A_{i}+\sum v_{i} B_{i} \preceq C\right\}$. We have

$$
\begin{aligned}
\alpha\left(\beta t_{1}+(1-\beta) t_{2}\right) & =\max _{(u, v) \in \Omega} u^{\top}\left(P Q^{g}+\left(\beta t_{1}+(1-\beta) t_{2}\right) \bar{d}\right)+v^{\top} c+r \\
& =\max _{(u, v) \in \Omega} \beta\left(u^{\top}\left(P Q^{g}+t_{1} \bar{d}\right)+v^{\top} c+r\right)+(1-\beta)\left(u^{\top}\left(P Q^{g}+t_{2} \bar{d}\right)+v^{\top} c+r\right) \\
& \leq \beta \alpha\left(t_{1}\right)+(1-\beta) \alpha\left(t_{2}\right),
\end{aligned}
$$

which leads to the convexity of $\alpha(t)$. For the second part, suppose the strong duality posited by Property 2 holds for $\left(\boldsymbol{S}^{s}\right)$ and (4) at the primal optimum $\left(\hat{P Q^{g}}, \hat{V}^{s}, \hat{P Q}{ }^{i n, s}, \hat{P Q}{ }^{\text {out }, s}\right)$ and dual optimum $(\hat{\lambda}, \hat{\gamma})$ respectively. We have

$$
\begin{aligned}
\omega^{s}\left(\hat{P Q^{g}}\right)+\hat{\gamma}^{\top} \hat{P Q^{g}} & =l(\hat{\lambda}, \hat{\gamma}) \\
& =g^{s}\left(\hat{P}^{i n, s}\right)-h^{s}\left(\hat{P}^{\text {out }, s}\right)+\hat{\lambda}^{\top} R^{s}\left(\hat{V}^{s}, \ldots\right)+\hat{\gamma}^{\top} E^{s}\left(\hat{V}^{s}\right) \\
& \leq g^{s}\left(P^{i n, s}\right)-h^{s}\left(P^{\text {out }, s}\right)+\hat{\lambda}^{\top} R^{s}\left(V^{s}, \ldots\right)+\hat{\gamma}^{\top} E^{s}\left(V^{s}\right) \\
& \leq \omega^{s}\left(P Q^{g}\right)+\hat{\gamma}^{\top} P Q^{g} .
\end{aligned}
$$

This completes the proof, since

$$
\omega^{s}\left(P Q^{g}\right) \geq \omega^{s}\left(\hat{P Q^{g}}\right)-\hat{\gamma}^{\top}\left(P Q^{g}-\hat{P Q^{g}}\right) .
$$

Theorem 1 tells us that the recourse function $\omega^{s}(\cdot)$ associated with the $s$-th subproblem $\left(\boldsymbol{S}^{\boldsymbol{s}}\right)$ is convex, and its dual optimal solution at a specific master-problem value $P Q^{g}$ provides a sub-gradient to the function $\omega^{s}(\cdot)$. This suggests that given a set of sub-gradients $\left\{\pi^{s, k}, k=1, \ldots, K\right\}$ of $\omega^{s}$, a piece-wise linear lower-approximation function $\eta^{s}$ can be constructed for $\omega^{s}$ that obeys a set of linear constraints of the form

$$
\omega^{s}\left(P Q^{g, k}\right)+\left(\pi^{s, k}\right)^{\top}\left(P Q^{g}-P Q^{g, k}\right) \leq \eta^{s}, \quad \forall k=1, \ldots, K .
$$

We exploit (5) to iteratively solve a sequence lower-approximation of the master problem (M) :

$$
\begin{array}{ll}
\min & \Psi^{k}\left(P Q^{g}\right)=f\left(P^{g}\right)+\sum p^{s} \eta^{s} \\
\text { subject to } & \left(P Q^{g}, V^{0}, P Q^{\text {in }, 0}, P Q^{\text {out }, 0}, P Q^{w, 0}\right) \in X
\end{array}
$$

and $\eta^{s}$ satisfies constraints of form (5),

where the $k$-th iteration yields sub-gradients $\pi^{s, k}$ centered around $P G^{g, k}$. The algorithm is as follows: 


\section{Phan and Ghosh}

Outer Approximation Algorithm 1

$$
\begin{aligned}
\text { 1. Set } \eta^{s, 1}=0 \forall s \in \mathcal{P} \text {. } & \text { Solve the following to set } P Q^{g, 1}=P^{g, 1}+\mathfrak{i} Q^{g, 1} \\
& \min f\left(P^{g}\right) \\
\text { s.t. } & \left(P Q^{g}, V^{0}, P Q^{i n, 0}, P Q^{\text {out }, 0}, P Q^{w, 0}\right) \in X .
\end{aligned}
$$

2. For $k=1,2, \ldots$

(a) For $s=1, \ldots,|\mathcal{P}|$ :

Solve the subproblem $\left(S^{s}\right)$ associated with $P Q^{g, k}=P^{g, k}+\mathfrak{i} Q^{g, k}$

to get the optimal value $\omega^{s}\left(P Q^{g, k}\right)$ and a subgradient $\pi^{s, k}$.

(b) Terminate the algorithm if

$$
\sum_{s} p^{s} \eta^{s, k}=\sum_{s} p^{s} \omega^{s}\left(P Q^{g, k}\right)
$$

(c) Solve the $k$-th lower-approximation $\left(\boldsymbol{M}^{k}\right)$ master problem to obtain an optimal solution $P Q^{g, k+1}=P^{g, k+1}+\mathfrak{i} Q^{g, k+1}$ and $\eta^{s, k+1}$.

In this algorithm, we add $|\mathcal{P}|$ new linear constraints per outer iteration. Notice that $\eta^{s, k}$ is the approximation of $\omega^{s}\left(P Q^{g, k}\right)$ given by the $k$-th piecewise-linear lower approximation from (5), and so $\sum_{s} p^{s} \eta^{s, k}=\sum_{s} p^{s} \omega^{s}\left(P Q^{g, k}\right)$ for every $k$. Thus, the algorithm terminates when the $k$-th master-problem finds an optimal $P G^{g}$ where the lower-approximation matches the true function $\omega^{s}$. Each master-problem iteration $\left(\boldsymbol{M}^{\boldsymbol{k}}\right)$ solves a classical optimal power flow problem augmented with a set of linear inequalities. Thus, $|\mathcal{P}|+1$ OPF problems are being solved per outer iteration each of which can be solved efficiently using the Lagrangian-dual-based algorithms presented in Phan (2010) or Lavaei and Low (2010). Under some mild conditions, we are also able to give a convergence analysis for this algorithm:

Theorem 2 Suppose that $\left\{\pi^{s, k}\right\}$ is uniformly bounded, i.e., there exists a constant $C$ such that $\left\|\pi^{s, k}\right\| \leq$ $C$ for every $s, k$, where $\|\cdot\|$ indicates the Euclidean norm. Then the Algorithm 1 either reaches an optimal solution in a finite number of iterations, or generates a sequence $\left\{\left(P Q^{g, k}\right)\right\}_{k=0, \ldots}$ such that

$$
\lim _{k \rightarrow \infty} \Psi\left(P Q^{g, k}\right)=\Psi^{*}
$$

where $\Psi^{*}$ is the optimal value of $(\mathbf{M})$.

Proof. If the algorithm terminates in a finite number of iterations, this implies that it stops at Step 2.b. We now show that $\Psi\left(P Q^{g, k+1}\right)=\Psi^{*}$ if Step 2.b is satisfied by $\left(P Q^{g, k+1}\right)$. We have

$$
\begin{aligned}
& f\left(P^{g, k+1}\right)+\sum_{s \in \mathcal{P}} \omega^{s}\left(P Q^{g, k+1}\right) p^{s} \\
& =f\left(P^{g, k+1}\right)+\sum_{s \in \mathcal{P}} \eta^{s,(k+1)} p^{s} \\
& \leq f\left(P^{g}\right)+\sum_{s \in \mathcal{P}} \max _{j=1, \ldots, k}\left\{\omega^{s}\left(P Q^{g, j}\right)+\left(\pi^{s, j}\right)^{\top}\left(P Q^{g}-P Q^{g, j}\right)\right\} p^{s} \\
& \leq f\left(P^{g}\right)+\sum_{s \in \mathcal{P}} \omega^{s}\left(P Q^{g}\right) p^{s},
\end{aligned}
$$

for every feasible point $\left(P Q^{g}\right)$ of problem $(\mathbf{M})$. The first inequality is due to the solution property of the master problem and the fact that

$$
\eta^{s, k+1}=\max _{j=1, \ldots, k}\left\{\omega^{s}\left(P Q^{g, j}\right)+\left(\pi^{s, j}\right)^{\top}\left(P Q^{g}-P Q^{g, j}\right)\right\} .
$$




\section{Phan and Ghosh}

The last inequality is because $\ell_{j}\left(P Q^{g}\right)=\omega^{s}\left(P Q^{g, j}\right)+\left(\pi^{s, j}\right)^{\top}\left(P Q^{g}-P Q^{g, j}\right)$ is an underestimate of $\omega^{s}\left(P Q^{g}\right)$ for every $j=1, \ldots, k$. The above relation concludes that $P Q^{g, k+1}$ is an optimal solution of (M).

Now suppose that $\left\{\left(P^{g, k}, Q^{g, k}\right)\right\}$ is an infinite sequence. We show that for any $\epsilon>0$, the set $I_{\epsilon}=\left\{k: \Psi^{*}<\Psi\left(P^{g, k}, Q^{g, k}\right)-\epsilon\right\}$ is finite. Let $k_{1}, k_{2} \in I_{\epsilon}$ and $k_{2}>k_{1}$. At $k_{1}$-th iteration, the optimality cut is defined by

$$
\omega^{s}\left(P Q^{g, k_{1}}\right)+\left(\pi^{s, k_{1}}\right)^{\top}\left(P Q^{g}-P Q^{g, k_{1}}\right) \leq \eta^{s} .
$$

Since $k_{2}>k_{1}$, plugging $\left(P Q^{g, k_{2}}, \eta^{s, k_{2}}\right)$ into (6) yields

$$
\begin{aligned}
& \sum_{s \in \mathcal{P}} \omega^{s}\left(P Q^{g, k_{1}}\right) p^{s}+\sum_{s \in \mathcal{P}}\left(\pi^{s, k_{1}}\right)^{\top}\left(P Q^{g, k_{2}}-P Q^{g, k_{1}}\right) p^{s} \leq \sum_{s \in \mathcal{P}} \eta^{s, k_{2}} p^{s} \\
& \Rightarrow \Psi\left(P Q^{g, k_{1}}\right)+\sum_{s \in \mathcal{P}}\left(\pi^{s, k_{1}}\right)^{\top}\left(P Q^{g, k_{2}}-P Q^{g, k_{1}}\right) p^{s}+f\left(P^{g, k_{2}}\right) \\
& \leq \sum_{s \in \mathcal{P}} \eta^{s, k_{2}} p^{s}+f\left(P^{g, k_{1}}\right)+f\left(P^{g, k_{2}}\right) \\
& \leq f\left(P^{g, k_{1}}\right)+\Psi^{*}
\end{aligned}
$$

since the optimal value of the master is a lower bound of $\Psi^{*}$. Hence

$$
\begin{aligned}
& -\Psi\left(P Q^{g, k_{1}}\right)-\sum_{s \in \mathcal{P}}\left(\pi^{s, k_{1}}\right)^{\top}\left(P Q^{g, k_{2}}-P Q^{g, k_{1}}\right) p^{s}-f\left(P^{g, k_{2}}\right)+f\left(P^{g, k_{1}}\right) \\
& \geq \Psi^{*}>\epsilon-\Psi\left(P Q^{g, k_{2}}\right), \text { since } k_{2} \in I_{\epsilon} .
\end{aligned}
$$

It follows that

$$
\sum_{s \in \mathcal{P}}\left(\omega^{s}\left(P Q^{g, k_{2}}\right)-\omega^{s}\left(P Q^{g, k_{1}}\right)-\left(\pi^{s, k_{1}}\right)^{\top}\left(P Q^{g, k_{2}}-P Q^{g, k_{1}}\right)\right) p^{s}>\epsilon .
$$

Note that $\left|\omega^{s}\left(P Q^{g, k_{2}}\right)-\omega^{s}\left(P Q^{g, k_{1}}\right)\right| \leq\left\|\pi^{s, k_{2}}\right\|\left\|P Q^{g, k_{2}}-P Q^{g, k_{1}}\right\|$. Combining these, we get

$$
\left\|P Q^{g, k_{2}}-P Q^{g, k_{1}}\right\|>\frac{2 C}{\max _{s \in \mathcal{P}} p^{s}} \epsilon
$$

for all $k_{1}, k_{2} \in I_{\epsilon}$. Since $\left\{P Q^{g, k}\right\}_{k=1, \ldots}$ is contained in a compact set, $I_{\epsilon}$ is finite.

By the aggregation of optimality cuts (5) for all scenarios, we can derive another cut

$$
\eta \triangleq \sum_{s} p^{s} \eta^{s} \geq \sum_{s \in \mathcal{P}} \omega^{s}\left(P Q^{g, k}\right) p^{s}+\sum_{s \in \mathcal{P}}\left(\pi^{s, k}\right)^{\top}\left(P Q^{g}-P Q^{g, k}\right) p^{s} .
$$

A variant $\left(\boldsymbol{M}^{\boldsymbol{k}^{\prime}}\right)$ of the lower-approximation problem $\left(\boldsymbol{M}^{\boldsymbol{k}}\right)$ where the variables $\left\{\eta^{s}\right\}$ are replaced by $\eta$ and the constraints (5) are replaced by constraints (7) is also a lower-approximation of the master problem (M). An alternate algorithm can be constructed by replacing $\left\{\eta^{s}\right\}$ and the lower-approximation problems $\left(\boldsymbol{M}^{k}\right)$ with the variable $\eta$ and the variant $\left(\boldsymbol{M}^{\boldsymbol{k}^{\prime}}\right)$. The algorithm is structured:

\section{Outer ApProximation Algorithm 2}




\section{Phan and Ghosh}

$$
\begin{aligned}
& \text { 1. Set } \eta^{1}=0 \forall s \in \mathcal{P} \text {. Solve the following to set } P Q^{g, 1}=P^{g, 1}+\mathfrak{i} Q^{g, 1} \\
& \qquad \begin{aligned}
\min & f\left(P^{g}\right) \\
& \left(P Q^{g}, V^{0}, P Q^{\text {in, }, 0}, P Q^{\text {out }, 0}, P Q^{w, 0}\right) \in X .
\end{aligned}
\end{aligned}
$$

2. $\operatorname{For} k=1,2, \ldots$

(a) For $s=1, \ldots,|\mathcal{P}|$ :

Solve the subproblem $\left(S^{s}\right)$ associated with $P Q^{g, k}=P^{g, k}+\mathfrak{i} Q^{g, k}$

to get the optimal value $\omega^{s}\left(P Q^{g, k}\right)$ and a subgradient $\pi^{s, k}$.

(b) Terminate the algorithm if

$$
\eta^{k}=\sum_{s} p^{s} \omega^{s}\left(P Q^{g, k}\right)
$$

(c) Solve the $k$-th lower-approximation $\left(\boldsymbol{M}^{\boldsymbol{k}^{\prime}}\right)$ master problem to obtain an optimal solution $P Q^{g, k+1}=P^{g, k+1}+\mathfrak{i} Q^{g, k+1}$ and $\eta^{k+1}$.

The approximating master problem from this algorithm is slightly different from the previous one. We add only one cut per major iteration, so its size is smaller. With a little modification of the arguments from the above proof, we can obtain a similar convergence theorem for this algorithm.

\section{NUMERICAL EXPERIMENTS}

We use the following test power systems to demonstrate the usefulness of our proposed algorithms. Their characteristics are summarized in Table 1.

- CH9: the 9 bus example from (Chow, Frederick, and Chbat 2002, p.70)

- NE39: the New England system (Pai 1989)

- IEEE14, IEEE30, IEEE57, IEEE118 and IEEE300: the five IEEE systems, they can be found at http://www.ee.washington.edu/research/pstca/

Table 1: Test systems characteristics.

\begin{tabular}{|l|c|c|c|c|c|c|}
\hline Test system & Buses & Generators & Lines & Wind generators & Export & Import \\
\hline CH9 & 9 & 3 & 9 & 4,6 & 5 & 2,3 \\
\hline IEEE14 & 14 & 5 & 20 & 4,6 & 5 & 2,3 \\
\hline IEEE30 & 30 & 6 & 41 & 4,6 & 5 & 2,3 \\
\hline IEEE57 & 57 & 7 & 80 & 5,45 & 7 & 3,4 \\
\hline IEEE118 & 118 & 54 & 186 & 89,100 & 60,75 & 25,65 \\
\hline IEEE300 & 300 & 69 & 411 & 89,100 & 60,75 & $2,89,150$ \\
\hline
\end{tabular}

The first column in Table 1 shows the abbreviations of the systems, while the second and third columns show the total number of buses and the number of generators in each system. The fourth column reports the number of lines interconnecting the buses. We added two sources of renewable generation, and chose their associated nodes, and those of the spot market locations, as presented in the remaining columns. The algorithms were coded in Matlab and experiments were carried out on a PC using Matlab 7.10 with an Intel Xeon X5570 $2.93 \mathrm{GHz}$ under the Linux operating system. We terminated the algorithms when $\left(\epsilon=10^{-3}\right)$

$$
\sum_{s \in \mathcal{P}} \omega^{s}\left(P Q^{g, k+1}\right) p^{s}-\sum_{s \in \mathcal{P}} \eta^{s, k+1} p^{s} \leq \epsilon \quad \text { (Algorithm 1) }
$$




\section{Phan and Ghosh}

and

$$
\sum_{s \in \mathcal{P}} \omega^{s}\left(P Q^{g, k+1}\right) p^{s}-\eta^{k+1} \leq \epsilon \quad \text { (Algorithm 2). }
$$

We set the mean power output of the first wind generator at $70 \%$ of the average of the upper limits $P^{\max }, Q^{\max }$ of the capacity of all conventional generators for the first wind generator. We sampled the second generator's mean uniformly around the first's mean within up to a $30 \%$ change. The cost coefficients for the imported cost function $g^{s}$ were randomly chosen from 1.5 to 2.0 times higher than the largest coefficients of the generation cost function $f$, i.e., we have to incur higher prices than our own generation cost if the energy has to be purchased from the spot market. Meanwhile, the coefficients of the exported cost function $h^{s}$ were taken by 0.5 to 0.7 times related to the smallest linear coefficients of $f$. Here, we assumed that $g^{s}$ and $h^{s}$ do not depend on $s$. The second stage scenarios were sampled uniformly with renewable generation varying up to $20 \%$ from their mean, which also represents their first-stage realized generation. In this manner, 20 scenarios of wind power outputs were chosen for each experimental run.

Table 2: The performance of algorithms. CPU time in seconds.

\begin{tabular}{|l|c|c|c|c|c|c|}
\hline \multicolumn{4}{|c|}{} & \multicolumn{2}{c|}{ Algorithm 1 } & \multicolumn{2}{c|}{ Algorithm 2 } \\
\hline Test system & Expected cost & Gen. cost & iter & time & iter & time \\
\hline CH9 & 2084.9 & 2053.8 & 2 & 0.426 & 2 & 0.418 \\
\hline IEEE14 & 5872.4 & 5785.9 & 2 & 0.481 & 2 & 0.475 \\
\hline IEEE30 & 6788.3 & 6695.7 & 4 & 1.706 & 4 & 1.657 \\
\hline NE39 & 32786.1 & 32794.1 & 2 & 0.798 & 2 & 0.708 \\
\hline IEEE57 & 37501.4 & 37331.7 & 5 & 0.469 & 7 & 0.675 \\
\hline IEEE118 & 128321.9 & 128115.8 & 4 & 3.659 & 4 & 3.306 \\
\hline IEEE300 & 714300.2 & 714030.3 & 3 & 5.048 & 3 & 4.932 \\
\hline
\end{tabular}

Table 2 shows that our proposed algorithms converge quickly to the optima, requiring from 2 to 7 iterations to satisfy the error tolerance. In most cases, the two algorithms used the same number of iterations to solve the problem. For these cases, the running time for Algorithm 2 is observed to be less than those of Algorithm 1. The sole exception is the IEEE 57 bus test system, for which Algorithm 2 requires more iterations, and as a result more computational time. Since the outer-approximation provided by the individual linear cuts from Algorithm 1 provides a tighter bound to $\omega^{s}($.$) , the required iterations never$ exceed those of Algorithm 2. On the other hand, Algorithm 2 uses fewer linear functions to provide the outer-approximation, and hence solvers take less time solving them.

We report in Figure 1(a) the effect of varying the maximum deviation allowed for the uncertainty of wind outputs on the total expected cost for the 57 bus test case. We use the average of 100 runs for each deviation. Figure 1(a) shows that as the deviation increases, so does the cost. Figure 1(b) plots the errors as defined on the left-hand side of (8) and (9) versus the number of iterations for two algorithms to solve the 57 bus test case.

\section{CONCLUSIONS}

This article proposes the first algorithms to solve a two-stage non-linear stochastic formulation for the economic dispatch problem faced by power transmission authorities under renewable-generation uncertainty. The structure of the formulation hews to standard two-stage problems, in that certain generation decisions are made only in the first stage and the second stage realizes the actual renewable generation, and the uncertainty is captured by a finite number of scenarios. Recourse for alleviating supply-demand mis-matches in the second stage is provided by high marginal-cost power sources that can be tapped in short order.

Our contributions to the literature lie in the novel outer approximation algorithms we propose to solve this non-convex optimization problem to optimality. We show that for problem instances that satisfy certain 


\section{Phan and Ghosh}

Figure 1: Total expected cost and performance progress for IEEE57.

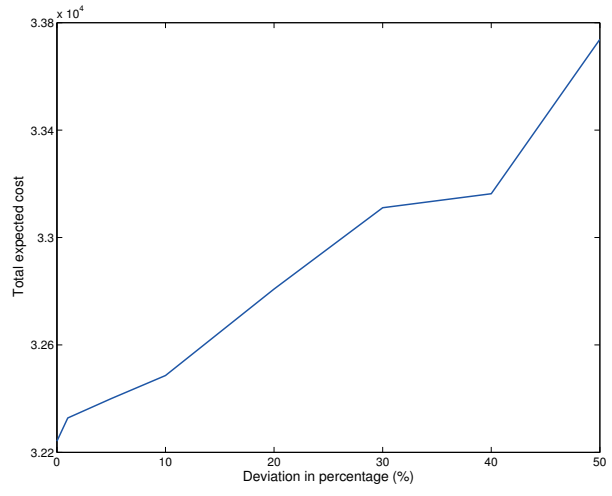

(a) Total Expected Cost vs Uncertainty Interval.

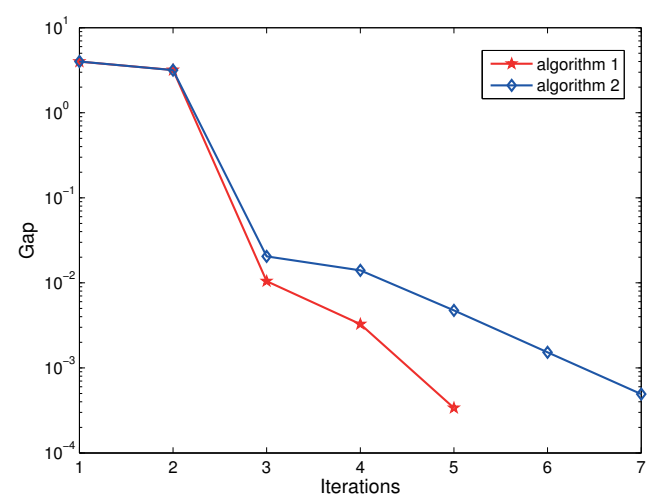

(b) Progress by Iteration.

conditions, an effective decomposition scheme can be used, just like in two-stage linear programs, to obtain a sequence of approximate solutions that has a limit point that is a globally-optimal solution to the two-stage non-convex program. In particular, this facilitates a parallel implementation of the solution algorithm, which we have not explored in the numerical experiments provide here. Our experiments for a variety of parameter settings indicate that the two approximation schemes we describe are efficient and usable even in large practical instances.

\section{ACKNOWLEDGMENTS}

This material is based upon work supported by the Department of Energy under Award Number DEOE0000190.

\section{REFERENCES}

Bonami, P., L. T. Biegler, A. R. Conn, G. Cornuéjols, I. E. Grossmann, C. D. Laird, J. Lee, A. Lodi, F. Margot, N. Sawaya, and A. Wëchter. 2008. "An algorithmic framework for convex mixed integer nonlinear programs". Discrete Optimization 5 (2): 186-204.

Boyd, S., and L. Vandenberghe. 2004. Convex optimization. Cambridge University Press.

Brini, S., H. H. Abdallah, and A. Ouali. 2009. "Economic Dispatch for Power System included Wind and Solar Thermal energy". Leonardo Journal of Sciences 14:204-220.

Carpentier, J. 1962. "Contribution to the economic dispatch problem". Bull Soc. France Elect. 8:431-447.

Cheung, K., X. Wang, B.-C. Chiu, Y. Xiao, and R. Rios-Zalapa. 2010, Jan.. "Generation dispatch in a smart grid environment”. In 2010 Innovative Smart Grid Technologies (ISGT), 1-6.

Chow, J., D. Frederick, and N. Chbat. 2002. Discrete-Time Control Problems Using MATLAB. CL Engg.

Dragoon, K., and M. Milligan. 2003. "Assessing wind integration costs with dispatch models: a case study of PacifiCorp". In Windpower 2003 Conference Proceedings (CD-ROM), Austin, TX.

Duran, M., and I. Grossmann. 1986. "An outer-approximation algorithm for a class of mixed-integer nonlinear programs". Math. Program. 36:307-339.

Fletcher, R., and S. Leyffer. 1994. "Solving mixed integer nonlinear programs by outer approximation". Math. Program. 66:327-349.

Fu, W., and J. D. McCalley. 2001, September. "Risk Based Optimal Power Flow". In 2001 IEEE Porto Power Tech Conference.

Geoffrion, A. M. 1972. “Generalized Benders decomposition”. J. Optim. Theory Appl. 10 (4): 237-260. 


\section{Phan and Ghosh}

Ghosh, S., J. R. Kalagnanam, D. Katz, M. S. Squillante, and X. Zhang. 2010. "Incentive Design for Lowest Cost Aggregate Energy Demand Reduction”. In Proceedings of First IEEE International Conference on Smart Grid Communications, 519-524.

Ghosh, S., J. R. Kalagnanam, D. Katz, M. S. Squillante, and X. Zhang. 2011. "Integration of Demand Response and Renewable Resources for Power Generation Management". In Proceedings of First IEEE Power \& Energy Society ISGT Meeting.

Grothey, A., S. Leyffer, and K. I. M. Mckinnon. 1999. "A note on feasibility in Benders Decomposition". University of Dundee Numerical Analysis Report NA-188.

Hatami, A. R., H. Seifi, and M. K. Sheikh-El-Eslami. 2009. "Hedging risks with interruptible load programs for a load serving entity". Decis. Support Syst. 48 (1): 150-157.

Lavaei, J., and S. Low. 2010, Oct.. "Convexification of Optimal Power Flow Problem". In Forty-Eighth Annual Allerton Conference on Communication, Control and Computing. Monticello, IL.

Li, H., Y. Li, and Z. Li. 2007. "A multiperiod energy acquisition model for a distribution company with distributed generation and interruptible load". IEEE Transactions on Power Systems 22 (2): 588-596.

Pai, M. 1989. Energy Function Analysis for Power System Stability. Boston, MA: Kluwer.

Phan, D. T. 2010. "Lagrangian duality and branch-and-bound algorithms for optimal power flow". (to appear in Operations Research).

Ruszczýnski, A. 2003. "Decomposition methods". In Handbook in Operations Research and Management Science, Volume on Stochastic Programming, Edited by A. Ruszczýnski and A. Shapiro, 141-211. Amsterdam: Elsevier.

Sahinidis, N., and I. Grossmann. 1991. "Convergence properties of generalized Benders decomposition". Computers \& Chemical Engineering 15 (7): 481-491.

Society, I. P. E. 1996. Optimal Power Flow: Solution Techniques, Requirements and Challenges. IEEE Tutorials.

Xue, Y., L. Chang, and J. Meng. 2007. "Dispatchable Distributed Generation Network - A New Concept to Advance DG Technologies". In 2007 IEEE Power Engineering Society General Meeting, 1-5.

\section{AUTHOR BIOGRAPHIES}

DZUNG PHAN is a Research Staff Member of the Mathematical Sciences Department at IBM T.J. Watson Research Center, Yorktown Heights, NY. His research interests lie in the field of the optimization theory and algorithms. Recently at IBM, he developed several numerical algorithms for optimization problems arising from power system analysis. He earned a $\mathrm{PhD}$ in Applied Mathematics from the University of Florida, a MS in Computational Engineering from the National University of Singapore, and a B.S. in Mathematics from Vietnam National University. His email address is phandu@us.ibm.com.

SOUMYADIP GHOSH is a Research Staff Member of the Mathematical Sciences Division at IBM T.J. Watson Research Center, Yorktown Heights, NY. His simulation research interests include theory and practice in particular on simulation-based optimization techniques and input dependence modeling and risk modeling. His other interests lie in the fields of smarter energy management, supply-chain analysis, and queueing theory based scheduling of large-scale production systems. He can be contacted at ghoshs@us.ibm.com and his web-page is https://researcher.ibm.com/researcher/view.php?person=us-ghoshs. 